\title{
Матриксные металлопротеиназы и их роль в развитии ремоделирования у больных, перенесших острый инфаркт миокарда (обзор литературы)
}

\author{
В.В. Букатов \\ ОГБУЗ «Белгородская областная клиническая больница Святителя Иоасафа», \\ Россия, 308007, г. Белгород, ул. Некрасова, 8/9 \\ E-mail: v.bukatoff@yandex.ru
}

\begin{abstract}
Аннотация. Особый интерес представляют собой механизмы ремоделирования миокарда после перенесенного инфаркта миокарда (ИМ). К сожалению, на сегодняшний день у науки нет однозначного ответа на вопрос о процессе ремоделирования миокарда после ИМ. Так, например, у двух схожих клинически пациентов, без сопутствующих заболеваний, с одинаковой локализацией ИМ, схожим временем открытия коронарной артерии в отдаленном периоде развивается совершенно разная картина сердечной недостаточности. У одного происходит дилатация полостей сердца, снижение фракции выброса, а у другого совершенно отсутствуют проявления сердечной недостаточности. Относительно недавно был установлен факт влияния матриксных металлопротеиназ на протекание ИМ, резкое повышение их концентрации в острый и отдаленный период наводят на рассуждения о их важнейшей роли в процессах ремоделирования миокарда. Настоящий обзор попытался обобщить полученные данные о роли матриксных металлопротеиназ и ИМ и выявить существующую закономерность.
\end{abstract}

Ключевые слова: инфаркт миокарда, ремоделирование миокарда, хроническая сердечная недостаточность, матриксные металлопротеиназы.

Для цитирования: Букатов В.В. 2020. Матриксные металлопротеиназы и их роль в развитии ремоделирования у больных, перенесших острый инфаркт миокарда (обзор литературы). Актуальные проблемы медицины, 43 (3): 392-403. DOI: 10.18413/2687-0940-2020-43-3-392-403

\section{Matrix metalloproteinases and their role in the development of remodeling in patients with acute myocardial infarction (review of literature)}

\author{
V.V. Bukatov \\ Belgorod Regional Clinical Hospital, \\ 8/9 Nekrasova St, Belgorod, 308007, Russia \\ E-mail: v.bukatoff@yandex.ru
}

\begin{abstract}
The mechanisms of myocardial remodeling after myocardial infarction (MI) are of particular interest. Unfortunately, today science does not have an unambiguous answer to the question about the process of myocardial remodeling after MI. For example, in two clinically similar patients, without concomitant diseases, with the same localization of myocardial infarction, with a similar opening time of the coronary artery, in the long-term period, a completely different picture of heart failure develops, one has dilatation of the heart cavities, a decrease in the ejection fraction, and the other has absolutely no manifestations heart failure. Relatively recently, the fact of the influence of matrix metalloproteinases on the course of myocardial infarction was established; a sharp increase in their concentration in the acute and long-term period suggests speculation about their most important role in the processes of myocardial remodeling. In this review, we tried to summarize the existing data on the role of matrix metalloprotenases and MI, and tried to identify the existing pattern.
\end{abstract}


Keywords: myocardial infarction, myocardial remodeling, chronic heart failure, matrix metalloproteinases.

For citation: Bukatov V.V. 2020. Matrix metalloproteinases and their role in the development of remodeling in patients with acute myocardial infarction (review of literature). Challenges in Modern Medicine, 43 (3): 392-403 (in Russian). DOI: 10.18413/2687-0940-2020-43-3-392-403

\section{Введение}

С тенденцией к росту продолжительности жизни также увеличивается количество больных с хронической сердечной недостаточностью $(\mathrm{XCH})$. В Российской Федерации (РФ) распространённость ХСН в промежуток с 1999 г. по 2014 г. выросла более чем в 2 раза, с 4,9\% до 10,2\%, причем распространенность тяжелых форм ХСН (функциональные классы III-IV) выросла более чем в 3 раза с 1,2\% до 4,1\% [Фомин, 2016]. Несмотря на то, что существует множество этиологичных факторов развития ХCH, ведущим остается ишемическая болезнь сердца (ИБС), а в частности такое ее проявление, как перенесенный ИМ. ИМ - это острое событие, которое развивается после длительного нарушения кровоснабжения, приводящего к необратимому повреждению ткани миокарда. Острый ИМ сопровождается прогрессирующим процессом заживления ран, который может перерасти в длительное патологическое ремоделирование ЛЖ. В последние несколько десятков лет, благодаря развитию науки в урбанизации населения, достигнут огромный прогресс в методах лечения ИМ. С развитием тромботической терапии чрезкожных коронарных вмешательств (ЧКВ) стало доступно, как казалось, этиологическое и патогенетическое лечение ИМ в виде быстрой реваскуляризации миокарда. Однако, как сказано выше, несмотря на достигнутый прогресс в лечении ИМ, процент больных с развитием тяжелых форм ХСН после перенесенного ИМ неуклонно растет. Основной причиной развития $\mathrm{XCH}$ после перенесенного ИМ является неправильное ремоделирование миокарда, формирование патологического рубца, дилатация полостей сердца и, как следствие, снижение фракции выброса (ФВ). Ремоделирование представляет собой длительной процесс, обусловленный сложным каскадом биохимических реакций, приводящий к формированию рубца и изменению здоровых миокардиоцитов.

Вышеописанный каскад биохимических реакций остается не до конца изученным. В последнее время появляются исследования, указывающие на важную роль матриксных металлопротеиназ (МПП) в процессах ремоделирования миокарда после перенесенного ИМ.

\section{Матриксные металлопротеиназы}

Матриксные металлопротеиназы (ММП) представляют собой семейство цинкзависимых эндопротеаз, выполняющих различные роли в процессах ремоделирования тканей и деградации множественных протеинов во внеклеточном матриксе. ММП способствуют пролиферации, миграции и дифференцировке клеток и могут играть роль в ангиогенезе, апоптозе клеток и репарации тканей. ММП также могут воздействовать на биоактивные молекулы на клеточной поверхности и модулировать различные клеточные и сигнальные пути. Изменения в экспрессии и активности ММП происходят в нормальных физиологических процессах, например, во время беременности и заживления ран, но также встречаются при сердечно-сосудистых заболеваниях, таких как атеросклероз, ИБС, аневризмы и хронические венозные заболевания [Khalil, 2018].

Семейство ММП имеет общую базовую структуру. Обычно ММП состоят из пропептида примерно из 80 аминокислот, каталитического металлопротеиназного домена примерно из 170 аминокислот, линкерного пептида или шарнирной области переменной длины и гемопексинового домена примерно из 200 аминокислот [Ohuchi et al., 1997]. 
Семейство матриксных металлопротеиназ разнообразно, и различные МПП влияют на различные мишени органов. В процессе ремоделирования миокарда, после перенесенного ИМ, наибольший интерес представляют собой ММП-8, 9.

После ИМ левый желудочек (ЛЖ) претерпевает ряд реакций, направленных на заживление сердечной раны, которые включают в себя как стимуляцию сильного воспаления для очистки погибших кардиомиоцитов и тканевого детрита, так и индукцию синтеза белка внеклеточного матрикса для стимуляции процессов рубцевания миокарда. Коллективные изменения в структуре и функции миокарда называются ремоделированием ЛЖ, а матричная металлопротеиназа-9 является ключевым инициатором ремоделирования ЛЖ после ИМ. Посредством прямого молекулярного воздействия на внеклеточный матрикс и метаболизм воспалительного белка, а также косвенного воздействия на основные типы клеток, которые координируют заживление сердечной раны, а именно инфильтрирующие лейкоциты и сердечные фиброасты, ММП-9 координирует множество аспектов ремоделирования ЛЖ [Rugmani et al., 2016].

ММП-9 регулирует ремоделирование левого желудочка после ИМ и тесно связана с воспалительным ответом на повреждение миокарда. Воспалительный ответ служит для рекрутирования лейкоцитов как части реакции заживления ран на повреждение миокарда, а инфильтрованные лейкоциты продуцируют цитокины и хемокины, которые стимулируют выработку и высвобождение ММП-9. В свою очередь, ММП-9 протеолизует цитокины и хемокины, в то время, как в большинстве случаев, расщепление ММП-9 субстрата цитокина или хемокина служит для увеличения активности воспалительной реакции. Существуют случаи, когда расщепление приводит к снижению активности последней. Глобальная делеция ММП-9 в моделях мышиного миокарда показала, что ингибирование некоторых аспектов активности ММП-9 может быть полезным для клинического применения. В то же время избыточная экспрессия ММП-9 в макрофагах в некоторых случаях указывала на положительные эффекты в процессах заживления миокарда, что указывает на то, что мы все еще не до конца понимаем сложность механизмов действия ММП-9 [DeleonPennell et al., 2015].

Инфильтрационные нейтрофилы и макрофаги являются надежным и ранним источником ММР-9 после ИМ как у людей, так и на экспериментальных животных моделях [Lindsey et al., 2001]. У мышей инфильтрация нейтрофилов происходит через несколько минут после ИМ, достигает пиков в первые трое суток и начинает уменьшаться к пятому дню. Полученный из нейтрофилов ММР-9 хранится в гранулах желатиназы и впоследствии высвобождается при хемотаксической стимуляции. К седьмому дню после ИМ число нейтрофилов возвращается к базовым значениям. Нейтрофилы в ишемическом миокарде генерируют интегриновые комплексы proММП-9/ $\alpha \mathrm{M} \beta 2$ во внутриклеточных вторичных гранулах, которые перемещаются на клеточную поверхность после активации. Блокирование взаимодействия между ММП-9 и $\alpha \mathrm{M} \beta 2$ ингибирует миграцию лейкоцитов in vivo и указывает на то, что ассоциация интегрина с ММП-9 способствует миграции клеток. ММП-9, полученный из нейтрофилов, оказывает ранние эффекты в условиях инфаркта миокарда, разрушая внеклеточный матрикс и способствуя проникновению макрофагальных клеток в область инфаркта [Zamilpa et al., 2012].

Существуют исследования, показывающие влияние ММП на иммунный ответ у пациентов с ИМ. Так, например, группа ученных во главе с Дж. Ангелини, Д. Флего [Angelini et al., 2018] доказала, что у пациентов с острым коронарным синдромом (ОКС) более высокая активность эффекторных Т-клеток. Это предполагает, что механизмы, включающие нарушение регуляции адаптивного иммунитета, могут играть роль в коронарной нестабильности. Отказ функционального домена 1-5 CD31 приводит к неконтролируемой активации лимфоцитов. В экспериментальных моделях матриксная ММП-9 участвует в эндотелиальном расщеплении CD31. Интересно, что при ОКС наблюдались более высокие сывороточные уровни ММП-9. Усиленное высвобождение ММП-9 играет 
ключевую роль в определении расщепления и высвобождения функционального домена CD31 1-5 в CD4 + T-клетках пациентов с ОКС. Этот механизм может представлять собой важную терапевтическую мишень для модуляции дисрегуляции Т-клеток при ОКС.

ММП-9 управляет многими аспектами воспалительной и пролиферативной стадий острого ИМ. Уровни ММП-9 повышаются очень рано после ИМ и остаются высокими в течение первой недели как на животных моделях ИМ, так и у людей с ИМ [Deten et al., 2002]. Раннее повышение уровня ММП-9 после ИМ коррелирует с повышенным числом лейкоцитов и размерами ЛЖ, а также с нарушением функции ЛЖ [DeLeon-Pennell et al., 2016]. Нацеленная делеция ММП-9 снижает количество макрофагов после ИМ, что приводит к ослаблению увеличения ЛЖ, предотвращает накопление коллагена, способствует неоваскуляризации и улучшает процессы ремоделирования ЛЖ. Интересно, что повышенная экспрессия трансгенной ММП-9 только в макрофагах неожиданно показала улучшение сердечной функции за счет стимуляции разрешения воспаления [Zamilpa et al., 2012].

Несмотря на вышеописанные данные, существует не так много клинических исследований, описывающих реальное влияние ММП-9 на процессы ремоделирования ЛЖ при остром ИМ. Д. Келли, Дж. Кокерил [Kelly et al., 2007] в своем исследовании показали прямую зависимость от высоких концентраций ММП-9 и более низкой фракции выброса (ФВ) ЛЖ во время госпитализации. Напротив, более высокие уровни плато ММП-9 были связаны с относительным сохранением функции ЛЖ и меньшим изменением конечного систолического и конечного диастолического объемов после выписки. Эти данные позволяют сделать неоднозначные выводы, что резкое повышение ММП-9 после острого ИМ связано со степенью ремоделирования ЛЖ. Напротив, более высокие уровни плато связаны с относительным сохранением функции ЛЖ. Возможно, более грамотно оценивать временной профиль, а не абсолютную величину активности ММП-9. Бланкенберг и его коллеги провели первое комплексное клиническое исследование, в котором ММП-9 использовался как новый прогностический биомаркер для лиц с повышенным риском смертности от сердечно-сосудистых заболеваний. ММП-9 коррелировал с белкамиреагентами острой фазы IL-6, hs-CRP и фибриногеном, что указывало на то, что ММП-9 может иметь собственное патофизиологическое значение в смертности от сердечнососудистых заболеваний. Сквайр и его коллеги расширили эти исследования, чтобы продемонстрировать, что у людей более высокий уровень ММП-9 коррелирует с большими объемами ЛЖ и большей дисфункцией после ИМ. Анализ пациентов из Фремингемского исследования обнаружил, что уровни ММП-9 в плазме связаны с увеличением диастолических размеров ЛЖ и увеличением толщины стенки. Доказано, что циркулирующие уровни ММП-9 независимо связаны с острым ИМ, но не со стабильной стенокардией. Уровни ММП-9 коррелировали с увеличением ЛЖ, снижением фракции выброса желудочков и стойким неблагоприятным ремоделированием ЛЖ у пациентов с хронической систолической сердечной недостаточностью. Фертин и др. изучили 112 корреляций между 52 различными биомаркерами и индексами ремоделирования ЛЖ. Наиболее устойчивые биомаркеры, связанные с ремоделированием ЛЖ, были связаны с нейрогормональной активацией. Среди биомаркеров ММП-9 пептиды коллагена и натрийуретический пептид Втипа были выдающимися биомаркерами, которые предсказывали неблагоприятное ремоделирование ЛЖ после ИМ.

Следует отметить, что несколько полиморфизмов были оценены в пределах гена ММП-9, и было показано, что они влияют на экспрессию гена, в частности, аллель С1562Т ассоциируется с повышенными концентрациями ММП-9 в плазме, тогда как полиморфизм R279Q не влияет на уровни в плазме, но ассоциируется с будущими сердечнососудистыми событиями. 279 аминокислот, в которых встречаются эти полиморфизмы, находятся в каталитическом домене фермента ММП-9 и позволяют предположить, что уровни активности ММП-9 могут быть выше у пациентов с полиморфизмом R279Q. B co- 
вокупности эти исследования предлагают убедительные доказательства важной роли ММП-9 в процессах ремоделирования ЛЖ.

В последние годы стало достоверно понятно влияние ММП-9 в ранних процессах ремоделирования. Однако стоит детально обратить внимание на влияние ММП-9 на процессы позднего ремоделирования миокарда. К сожалению, в настоящее время существует крайне мало научных исследований о влиянии ММП-9 на позднее ремоделирование миокарда.

П. Эндрю и коллеги [Voorhees et al., 2015] доказали, что понимание механических свойств ткани инфаркта важно для объяснения механизмов исходов после ИМ. Слишком эластичная ткань инфаркта может привести к расширению ЛЖ и даже к разрыву сердца, а слишком жесткая ткань, напротив, может привести к развитию диастолической сердечной недостаточности. Механическая прочность и жесткость коллагена обусловлены правильной сборкой волокон и сшивками. Мутация в гене коллагена $\alpha 2$ (I) у мышей препятствует правильной сборке коллагена I и приводит к увеличению дилатации ЛЖ и разрыву после ИМ. Образование ковалентных поперечных связей, стабилизирующих коллагеновые волокна, в первую очередь катализируются лизилоксидазой. Сама лизилоксидаза активируется протеазой костного морфогенетического белка-1, который также отщепляет Сконцевой пептид от проколлагена. Исследование показало, что делеция ММП-9 значительно увеличивает количество активированной лизилоксидазы в инфаркте миокарда на 7й день после ИМ и значительно снижает количество неактивной лизилоксидазы. Протеолитической активации лизилоксидазы костным морфогенетическим белком-1 способствует фибронектин, который служит каркасом для сборки коллагеновых волокон [Ben Fogelgren et al., 2005]. Фибронектин является субстратом для расщепления ММП-9, и уменьшение его фрагментации, вероятно, улучшает активацию лизилоксидазы костным морфогенетическим белком-1 и, в свою очередь, сборку коллагеновых волокон ММП-9.

Основываясь как на этом исследовании, так и на предыдущих выводах, делеция MМП-9 снижает общий фиброзный ответ за счет нескольких механизмов [Zamilpa et al., 2010]. Фиброзный ответ обычно действует как система отрицательной обратной связи с усилением фиброза, возникающим в ответ на усиление распада внеклеточного матрикса. Фрагменты внеклеточного матрикса, такие как фибронектин или фрагменты коллагена, связываются с рецепторами клеточной поверхности и вызывают усиление фиброза [Barak Marom et al., 2007]. Делеция ММП-9 ограничивает образование фрагментов фибронектина, что может объяснить уменьшение степени фиброза. Изучено, что изменения в тканевом матриксе модулируют синтез коллагена, при этом повышенные уровни стресса вызывают усиленное отложение коллагена [Rouillard, Holmes, 2012]. Согласно закону Лапласа, увеличенный объем ЛЖ и уменьшение толщины стенки, должны приводить к увеличению напряжения ЛЖ, что объясняет усиление фиброза после ИМ. Другая возможность заключается в повышенном ангиогенезе. Было показано, что делеция ММП-9 способствует образованию кровеносных сосудов, что может снизить уровень гипоксического стресса, дополнительно уменьшить фиброз и сохранить области пограничной зоны. Вероятно, именно сочетание этих механизмов приводит к уменьшению фиброза.

Взаимосвязь между качеством миокардиального рубца и его механическими свойствами во время острых стадий после инфаркта миокарда сложно определить отчасти изза быстрых изменений вовлеченных популяций клеток и молекулярного состава левого желудочка в ответ на ишемический инфаркт. Было бы важно знать, предотвращает ли делеция ММП-9 как дилатацию в ранние моменты времени, так и предотвращает ли диастолическую дисфункцию в более поздние моменты времени за счет уменьшения фиброза.

Матричная металлопротеиназа-9 играет решающую роль в разрыве атеросклеротической бляшки и ремоделировании ткани после сердечного приступа. Баланс между ММП-9 и эндогенным ингибитором, тканевыми ингибиторами матриксной металлопротеиназы 1 (ТIMР-1) важен при остром коронарном синдроме. 


\section{Тканевой ингибитор матриксных металопротеиназ 1 (ТИМП-1)}

ТИМП представляют собой низкомолекулярные белки, основное действие которых заключается в ингибировании протеолитической активности ММП путем связывания с активным доменом. Однако ТИМП обладают дополнительными биологическими действиями, в некоторой степени независимыми от их действий по ингибированию активности MMП [Webb et al., 2006]. Эти действия включают стимулирование роста и ингибирование как запрограммированной гибели клеток, так и ангиогенеза, и появляется все больше доказательств роли ТИМП-1, в частности в поддержании структуры и функции ЛЖ.

Д. Келли с коллегами [Kelly et al., 2006] установили четкую связь между циркулирующим ТИМП-1 и степенью дисфункции ЛЖ после ИМ. Показана дополнительная связь ТИМП-1 с эхокардиографическими маркерами размеров и дисфункции ЛЖ. Эти ассоциации были линейными, проявлялись в первые несколько дней после ИМ и сохранялись несколько недель спустя.

Установлен более высокий ТИМП-1 у пациентов мужского пола, курильщиков и лиц с гипертонией в анамнезе, что позволяет предположить, что ТИМП-1 в плазме может быть маркером неблагоприятного ремоделирования после перенесенного ИМ. Однако нельзя исключить, что более высокие концентрации ТИМП-1 в плазме могут представлять физиологический ответ на повышенную активность ММП и как таковые отражать попытку регулировать чрезмерную протеолитическую активность.

M.В. Голдбергова с коллегами [Goldbergova et al., 2012] оценили связь между уровнями ТИМП-1 и дисфункцией ЛЖ после перенесенного ИМ. Обнаружена взаимосвязь между более высокими уровнями ТИМП-1 через 24 часа после начала ИМ и низкой ФВ. Об аналогичных результатах отрицательной корреляции между уровнем ТИМП-1 и ФВ по данным догоспитальной эхокардиографии при выписке сообщили Д. Келли с коллегами [Kelly et al., 2006]. Существуют данные об отрицательной корреляции между уровнем ТИМП-1 через 24 часа и ФВ по данным магнитной резонансной томографии сердца, оцененной на пятый день и через четыре месяца после ИМ. Не обнаружена взаимосвязь между плазменными концентрациями ТИМП-1 и долгосрочным ремоделированием ЛЖ после ИМ. К сожалению, все эти клинические исследования не смогли продемонстрировать, что пациенты должны получать пользу от более высоких уровней ТИМП-1. Основная причина, вероятно, заключается в том, что значение ТИМП-1 в клинических условиях коррелирует с размером инфаркта. Более того, повышение уровня ТИМП-1 в течение 24 часов может быть вызвано реперфузионным повреждением миокарда после ЧКВ. Интересная информация заключается в том, что на концентрацию ТИМП-1 влияет предыдущее лечение статинами и бета-блокаторами. Было продемонстрировано, что статины стимулировали ТИМП-1, который впоследствии ингибировал ММП-1, -3, -9 и -13 Также было установлено, что аспирация тромба во время острого ИМ может приводить к снижению уровней циркулирующего ТИМП-1. Улучшение микроциркуляции с возможным повреждением нижних отделов миокарда после аспирации тромба может быть механизмом, ответственным за этот результат.

\section{ММП-8}

Как и большинство ММП, ММП-8 секретируется как неактивный профермент, который необходимо активировать, прежде чем он сможет выполнять свою функцию. Активация ММП-8 может быть опосредована реактивными формами кислорода, высвобождаемыми из активированных нейтрофилов, или различными протеазами, такими как катепсин $\mathrm{G}$, химотрипсин или ММП (-3, -7, -10 и -14). Это говорит о том, что активация ММП-8 действительно сильно регулируется и в основном ограничивается участками воспаления, чем системным кровотоком. После активации ММП-8 может расщеплять широкий спектр субстратов. В частности, ММП-8 разрушает коллаген I типа и является основным компо- 
нентом фиброзной покрышки, который защищает сосуд разрыва и поддерживает целостность стенки атеросклеротического сосуда в несколько раз сильнее, чем ММП-1 [Knäuper et al., 2006].

Долгое время считалось, что одна из самых интригующих ММП, ММП-8, также известная как коллагеназа-2 или коллагеназа нейтрофилов, экспрессируется исключительно в предшественниках нейтрофилов, поскольку она была клонирована из РНК, выделенной из лейкоцитов периферической крови пациента с хроническим гранулоцитарным лейкозом [Naruko et al., 2002]. В 2001 году Герман и его коллеги продемонстрировали, что ММП-8 также экспрессируется эндотелиальными клетками, гладкомышечными клетками и макрофагами в атеросклеротических поражениях человека, тем самым подтверждая, что экспрессия ММП-8 выходит за рамки одного типа. Действительно, авторы демонстрируют, что синтез и высвобождение ММП-8 требует длительного воздействия воспалительных цитокинов (таких как IL- $\beta$ или CD40L), тогда как нейтрофилы накапливают зимоген ММП-8 во внутриклеточных гранулах и высвобождают коллагеназы практически сразу после стимуляции.

К сожалению, концентрации ММП-8 в сыворотке и плазме редко определялись в контексте сердечно-сосудистых заболеваний (СС3), поэтому возможная связь между уровнями циркулирующего ММП-8 и сердечно-сосудистым риском еще предстоит оценить. Недавно были опубликованы результаты когорты пациентов, перенесших каротидную эндартерэктомию ( $=84)$, по поводу симптоматического и бессимптомного заболевания [Turu et al., 2006]. Авторы продемонстрировали, что уровни ММП-8 в плазме были значительно выше у пациентов с нестабильными гипоэхогенными бляшками по оценке ультразвукового исследования, чем у пациентов со стабильными гиперэхогенными бляшками. Другое исследование было проведено у пациентов, перенесших коронарную ангиографию (n = 250) по поводу ишемической болезни сердца (ИБС) [Molloy et al., 2004]. Концентрации ММП-8 в плазме были значительно выше у пациентов с ИБС по сравнению с таковыми у субъектов без заболевания. Более того, авторы наблюдали ступенчатое увеличение концентрации ММП-8 в зависимости от количества стенозированных коронарных артерий у пациентов с нестабильной стенокардией $(\mathrm{n}=45)$ и со стабильной ИБС $(\mathrm{n}=175)$. Момияма с соавторами обнаружили, что уровни ММП-8 в плазме были выше у пациентов со стабильной ИБС, чем у пациентов из контрольной группы. Они также заметили, что уровни ММП-8 у пациентов с нестабильной стенокардией были намного выше, чем у пациентов со стабильной стенокардией [Momiyama et al., 2010]. Совсем недавно в двух исследованиях случай-контроль ( $\mathrm{n}=141$ и 343 соответственно) высокие сывороточные концентрации ММП-8 и ММП-8/ТИМП-1 были тесно связаны с острым коронарным синдромом [Alfakry et al., 2010]. Таким образом, результаты, полученные в этих «больших» исследованиях, показали, что концентрации ММП-8 в плазме у пациентов с прогрессирующим атеросклерозом положительно связаны с наличием и тяжестью прогрессирования бляшек сонной артерии.

Решающий шаг был сделан Tuomainen и соавторами в проспективном исследовании на мужской популяции $(\mathrm{n}=1018)$ с клиническим наблюдением в течение 10 лет [Tuomainen et al., 2007]. Авторы показали, что концентрация ММП-8 в сыворотке крови является независимым фактором риска острого инфаркта миокарда, ИБС и ССЗ. Повышенный риск смерти от сердечно-сосудистых заболеваний был особенно значительным у пациентов мужского пола с субклиническим атеросклерозом на исходном уровне. Действительно, высокая концентрация ММП-8 в сыворотке крови увеличивала в 3 раза риск смерти от сердечно-сосудистых заболеваний во время наблюдения независимо от других известных факторов риска сердечно-сосудистых заболеваний. Однако необходимы дальнейшие анализы, чтобы подтвердить клиническую значимость ММП-8 при сердечнососудистых заболеваниях в общей популяции, включая женщин. 
Настоящие данные о циркулирующей ММП-8 могут иметь практическое значение как для диагностики, так и для лечения каротидных и коронарных заболеваний. Очевидно, что требуется гораздо больше исследований, прежде чем уровни ММП-8 в сыворотке могут быть валидированы как клинически полезный маркер для лучшей оценки риска острых сердечно-сосудистых событий.

\section{Заключение}

В настоящее время остаются открытыми достаточно много вопросов, касающихся непосредственного участия МПП в процессах ремоделирования миокарда. Остаются не выясненными отличительные особенности патогенетических механизмов формирования ремоделирования миокарда и их зависимость от степени повышения маркеров фиброза, а также структурно-функциональных, гемодинамических показателей сердца у пациентов с ИМ. Открыт вопрос о зависимости прогрессирования ХСН у пациентов геронтологической группы и влияние МПП на прогноз у этих пациентов.

ММП-9,8, ТИМП оказывают как отрицательные, так и положительные эффекты на ЛЖ после ИМ. Таким образом, любой вид терапевтического вмешательства, направленного на ММП-9, должен быть тщательно оценен. Есть несколько областей, в которых требуются дополнительные исследования in vivo и in vitro для более полного понимания функций ММП-9. Взаимодействие между временными и пространственными эффектами на экспрессию ММП-9 остается не совсем понятным. Взаимодействие ММП-9 с другими ММП еще предстоит изучить с точки зрения конкуренции ММП за конкретный субстрат. Точно так же проявляет ли ММП-9 предпочтение субстратов, когда присутствует множество субстратов, не оценивали с помощью конкурентного анализа. Более полное понимание субстратов ММП-9 необходимо для того, чтобы знать, какие субстраты предпочтительно расщепляются в условиях после ИМ и какова биологическая функция пептидов субстратов, генерируемых ММП-9.

\section{Список литературы}

1. Фомин И.В. 2016. Хроническая сердечная недостаточность в Российской Федерации: что сегодня мы знаем и что должны делать. Российский кардиологический журнал. 8: 7-13.

2. A. Deten, H.C. Volz, W. Briest, H. Zimmer. 2002. Cardiac cytokine expression is upregulated in the acute phase after myocardial infarction. Experimental studies in rats. Cardiovasc Res. 55: $329-340$

3. Andrew D. Rouillard, Jeffrey W. Holmes. 2012. Mechanical regulation of fibroblast migration and collagen remodeling in healing myocardial infarcts. The Journal of Physiology. 15; 590 (18): 4585-4602. doi: 10.1113/jphysiol.2012.229484.

4. Andrew P. Voorhees, Kristine Y. DeLeon-Pennell, Yonggang Ma, Ganesh V. Halade, Andriy Yabluchanskiy, Rugmani Padmanabhan Iyer, Elizabeth Flynn, Courtney A. Cates , Merry L. Lindsey, Hai-Chao Han. 2015. Building a Better Infarct: Modulation of Collagen Cross-linking to Increase Infarct Stiffness and Reduce Left Ventricular Dilation post-Myocardial Infarction. J. Mol. Cell. Cardiol. 85: 229-239. doi:10.1016/j.yjmcc.2015.06.006.

5. Anita M. Tuomainen, Kristiina Nyyssönen, Jari A. Laukkanen, Taina Tervahartiala, TomiPekka Tuomainen, Jukka T. Salonen, Timo Sorsa, Pirkko J. Pussinen. 2007. Matrix metalloproteinase-8 concentrations are associated with cardiovascular outcome in men. Serum Arteriosclerosis, Thrombosis, and Vascular Biology. 27 (12): 2722-272. doi: 10.1161/ATVBAHA.107.154831.

6. Barak Marom, Michal A., Rahat Nitza Lahat Lea Weiss Cerem, Amalia Kinarty, Haim Bitterman. 2007. Native and fragmented fibronectin oppositely modulate monocyte secretion of MMP-9. Journal of Leukocyte Biology. 81: 1466-1476.

7. Ben Fogelgren, Noémi Polgár, Kornélia Molnárné Szauter, Zsuzsanna Ujfaludi, Rozália Laczkó, Keith S. K. Fong, Katalin Csiszar. 2005. Cellular Fibronectin Binds to Lysyl Oxidase with High Affinity and Is Critical for Its Proteolytic Activation. Journal of Biological Chemistry. 280: 2469024697. 
8. Carson S. Webb, David D. Bonnema, S. Hinan Ahmed, Amy H. Leonardi, Catherine D. McClure, Leslie L. Clark, Robert E. Stroud, William C. Corn, Laura Finklea, Michael R. Zile, Francis G. Spinale. 2006. Specific temporal profile of matrix metalloproteinase release occurs in patients after myocardial infarction: relation to left ventricular remodeling. Circulation. 114: 1020-1027. doi: 10.1161/CIRCULATIONAHA.105.600353.

9. Dominic Kelly, Gillian Cockerill, Leong L. Ng, Matt Thompson, Sohail Khan, Nilesh J. Samani, Iain B. Squire. 2007. Plasma matrix metalloproteinase-9 and left ventricular remodelling after acute myocardial infarction in man: a prospective cohort study. Eur. Heart. J. Mar. 28 (6): 711-718. doi:10.1093/eurheartj/ehm003.

10. Dominic Kelly, Sohail Q. Khan, Matt Thompson, Gillian Cockerill, Leong L. Ng, Nilesh Samani, Lain B. Squire. Plasma tissue inhibitor of metalloproteinase-1 and matrix metalloproteinase9.2008. Novel indicators of left ventricular remodelling and prognosis after acute myocardial infarction. Eur Heart J. 29 (17): 2116-2124. doi: 10.1093/eurheartj/ehn315.

11. Giulia Angelini, Davide Flego, Ramona Vinci, Daniela Pedicino, Francesco Trotta, Aureliano Ruggio, Giuseppe P. Piemontese, Domenico Galante, Myriana Ponzo, Luigi M. Biasucci, Giovanna Liuzzo, Filippo Crea. 2018. Matrix metalloproteinase-9 might affect adaptive immunity in non-ST segment elevation acute coronary syndromes by increasing CD31 cleavage on CD4+ T-cells. Eur. Heart. J. 39(13): 1089-1097. doi: 10.1093/eurheartj/ehx684.

12. Hatem Alfakry, Juha Sinisalo, Susanna Paju, Markku S. Nieminen, Ville Valtonen, Taina Tervahartiala, Pirkko J. Pussinen, Timo Sorsa. 2012. The association of serum neutrophil markers and acute coronary syndrome. Scandinavian Journal of Immunology. 76 (2): 181-187. doi: 10.1111/j.13653083.2012.02718.x.

13. K. J. Molloy, M. M. Thompson, J. L. Jones, E. C. Schwalbe, P. R. F. Bell, A. R .Naylor, I. M. Loftus. 2004. Unstable carotid plaques exhibit raised matrix metalloproteinase-8. Circulation. 110 (3): 337-340 doi: 10.1161/01.CIR.0000135588.65188.14.

14. Khalil A. 2018. Matrix Metalloproteinases, Vascular Remodeling, and Vascular Disease Advances in Pharmacology. 81: 241-330. DOI: 10.1016/bs.apha.2017.08.002.

15. Kristine Y. Deleon-Pennell, Raffaele Altara, Andriy Yabluchanskiy, Alessandra Modesti, Merry L. Lindsey. 2015. The Circular Relationship between Matrix Metalloproteinase (MMP)-9 and Inflammation following Myocardial Infarction. IUBMB Life.67 (8): 611-618. doi: 10.1002/iub.1408.

16. Kristine Y. DeLeon-Pennell, Yuan Tian, Bai Zhang, Courtney A. Cates, Rugmani Padmanabhan Iyer, Presley Cannon, Punit Shah, Paul Aiyetan, Ganesh V. Halade, Yonggang Ma, Elizabeth Flynn, Zhen Zhang, Yu-Fang Jin, Hui Zhang, Merry L. Lindsey. 2016. CD36 is a matrix metalloproteinase-9 substrate that stimulates neutrophil apoptosis and removal during cardiac remodeling. Circ. Cardiovasc. Genet. 9: 14-25.

17. M. Lindsey, K. Wedin, M. D. Brown, C. Keller, A. J. Evans, J. Smolen, A. R. Burns, R. D. Rossen, L. Michael, M. Entman. 2001. Matrix-dependent mechanism of neutrophil-mediated release and activation of matrix metalloproteinase 9 in myocardial ischemia/reperfusion. Circulation. 103:2181-2187.

18. Marta Miguel Turu, Jerzy Krupinski, Esther Catena, Ana Rosell, Joan Montaner, Francisco Rubio, Jose Alvarez-Sabin, Marc Cairols, Lina Badimon. 2006. Intraplaque MMP-8 levels are increased in asymptomatic patients with carotid plaque progression on ultrasound. Atherosclerosis. 187 (1): 161-169. doi: 10.1016/j.atherosclerosis.2005.08.039.

19. Monika Pavkova Goldbergova, Jiri Parenica, Jiri Jarkovsky, Petr Kala, Martin Poloczek, Jan Manousek, Krystyna Kluz, Lenka Kubkova, Simona Littnerova, Martin Tesak, Ondrej Toman, Nikolas Pavek, Zdenka Cermakova, Josef Tomandl, Anna Vasku, Jindrich Spinar. 2012. The Association Between Levels of Tissue Inhibitor of Metalloproteinase-1 with Acute Heart Failure and Left Ventricular Dysfunction in Patients with ST Elevation Myocardial Infarction Treated by Primary Percutaneous Coronary Intervention. Genet. Test. Mol. Biomarkers.16 (10): 1172-1178. doi:10.1089/gtmb.2012.0120.

20. Ohuchi E., Imai K., Fujii Y., Sato H., Seiki M,. Okada Y. 1997. Membrane type 1 matrix metalloproteinase digests interstitial collagens and other extracellular matrix macromolecules. Biol Chem 24; 272(4) 2446-2451. doi: 10.1074/jbc.272.4.2446.

21. Rogelio Zamilpa, Elizabeth F. Lopez, Ying Ann Chiao, Qiuxia Dai, Gladys P. Escobar, Kevin Hakala, Susan T. Weintraub, Merry L. Lindsey. 2010. Proteomic analysis identifies in vivo candidate 
matrix metalloproteinase-9 substrates in the left ventricle post-myocardial infarction. Proteomics. 10: 2214-2223.

22. Rogelio Zamilpa, Jessica Ibarra, Lisandra E. de Castro Brás, Trevi A. Ramirez, Nguyen Nguyen, Ganesh V. Halade, Jianhua Zhang, Qiuxia Dai, Tariq Dayah, Ying Ann Chiao, Wesley Lowell, Seema S. Ahuja, Jeanine D'Armiento, Yu-Fang Jin, Merry L. Lindsey. 2012. Transgenic overexpression of matrix metalloproteinase-9 in macrophages attenuates the inflammatory response and improves left ventricular function post-myocardial infarction. J. Mol. Cell. Cardiol. 53: 599-608.

23. Rogelio Zamilpa, Jessica Ibarra, Lisandra E. de Castro Brás, Trevi A. Ramirez, Nguyen Nguyen, Ganesh V. Halade, Jianhua Zhang, Qiuxia Dai, Tariq Dayah, Ying Ann Chiao, Wesley Lowell, Seema S. Ahuja, Jeanine D’Armiento, Yu-Fang Jin, and Merry L. Lindsey, Ph.D. 2012. Transgenic overexpression of matrix metalloproteinase-9 in macrophages attenuates the inflammatory response and improves left ventricular function post-myocardial infarction. J. Mol. Cell. Cardiol. 53: 599-608.

24. Rugmani Padmanabhan Iyer, Mira Jung, Merry L. Lindsey. 2016. MMP-9 signaling in the left ventricle following myocardial infarction. Am. J. Physiol. Heart. Circ. Physiol. 311 (1): 190-198. doi: 10.1152/ajpheart.00243.

25. Takahiko Naruko, Makiko Ueda, Kazuo Haze, Allard C. van der Wal, Chris M. van der Loos, Akira Itoh, Ryushi Komatsu, Yoshihiro Ikura, Masayuki Ogami, Yoshihisa Shimada, Shoichi Ehara, Minoru Yoshiyama, Kazuhide Takeuchi, Junichi Yoshikawa, Anton E. Becker. 2002. Neutrophil infiltration of culprit lesions in acute coronary syndromes. Circulation. 106 (23): 2894-2900. doi: 10.1161/01.cir.0000042674.89762.20.

26. V. Knäuper, G. Murphy, H. Tschesche. 1996. Activation of human neutrophil procollagenase by stromelysin 2. European Journal of Biochemistry. 235 (1-2): 187-191.

27. Yukihiko Momiyama, Reiko Ohmori, Nobukiyo Tanaka, Ryuichi Kato, Hiroaki Taniguchi, Takeshi Adachi, Haruo Nakamura, Fumitaka Ohsuzu. 2010. High plasma levels of matrix metalloproteinase-8 in patients with unstable angina. Atherosclerosis. 209 (1): 206-210. DOI:https://doi.org/10.1016/j.atherosclerosis.2009.07.037.

\section{References}

1. Fomin I.V. 2016. Hronicheskaja serdechnaja nedostatochnost' v Rossijskoj Federacii: chto segodnja my znaem i chto dolzhny delat' [Chronic heart failure in the Russian Federation: what we know today and what we must do]. Rossijskij kardiologicheskij zhurnal. 8: 7-13.

2. A. Deten, H.C. Volz, W. Briest, H. Zimmer. 2002. Cardiac cytokine expression is upregulated in the acute phase after myocardial infarction. Experimental studies in rats. Cardiovasc Res. 55: 329-340

3. Andrew D. Rouillard, Jeffrey W. Holmes. 2012. Mechanical regulation of fibroblast migration and collagen remodeling in healing myocardial infarcts. The Journal of Physiology. 15; 590 (18): 4585-4602. doi: 10.1113/jphysiol.2012.229484.

4. Andrew P. Voorhees, Kristine Y. DeLeon-Pennell, Yonggang Ma, Ganesh V. Halade, Andriy Yabluchanskiy, Rugmani Padmanabhan Iyer, Elizabeth Flynn, Courtney A. Cates , Merry L. Lindsey, Hai-Chao Han. 2015. Building a Better Infarct: Modulation of Collagen Cross-linking to Increase Infarct Stiffness and Reduce Left Ventricular Dilation post-Myocardial Infarction. J. Mol. Cell. Cardiol. 85: 229-239. doi:10.1016/j.yjmcc.2015.06.006.

5. Anita M. Tuomainen, Kristiina Nyyssönen, Jari A. Laukkanen, Taina Tervahartiala, TomiPekka Tuomainen, Jukka T. Salonen, Timo Sorsa, Pirkko J. Pussinen. 2007. Matrix metalloproteinase-8 concentrations are associated with cardiovascular outcome in men. Serum Arteriosclerosis, Thrombosis, and Vascular Biology. 27 (12): 2722-272. doi: 10.1161/ATVBAHA.107.154831.

6. Barak Marom, Michal A., Rahat Nitza Lahat Lea Weiss Cerem, Amalia Kinarty, Haim Bitterman. 2007. Native and fragmented fibronectin oppositely modulate monocyte secretion of MMP-9. Journal of Leukocyte Biology. 81: 1466-1476.

7. Ben Fogelgren, Noémi Polgár, Kornélia Molnárné Szauter, Zsuzsanna Ujfaludi, Rozália Laczkó, Keith S. K. Fong, Katalin Csiszar. 2005. Cellular Fibronectin Binds to Lysyl Oxidase with High Affinity and Is Critical for Its Proteolytic Activation. Journal of Biological Chemistry. 280: 2469024697.

8. Carson S. Webb, David D. Bonnema, S. Hinan Ahmed, Amy H. Leonardi, Catherine D. McClure, Leslie L. Clark, Robert E. Stroud, William C. Corn, Laura Finklea, Michael R. Zile, Francis G. 
Spinale. 2006. Specific temporal profile of matrix metalloproteinase release occurs in patients after myocardial infarction: relation to left ventricular remodeling. Circulation. 114: 1020-1027. doi: 10.1161/CIRCULATIONAHA.105.600353.

9. Dominic Kelly, Gillian Cockerill, Leong L. Ng, Matt Thompson, Sohail Khan, Nilesh J. Samani, Iain B. Squire. 2007. Plasma matrix metalloproteinase-9 and left ventricular remodelling after acute myocardial infarction in man: a prospective cohort study. Eur. Heart. J. Mar. 28 (6): 711-718. doi:10.1093/eurheartj/ehm003.

10. Dominic Kelly, Sohail Q. Khan, Matt Thompson, Gillian Cockerill, Leong L. Ng, Nilesh Samani, Lain B. Squire. Plasma tissue inhibitor of metalloproteinase-1 and matrix metalloproteinase9.2008. Novel indicators of left ventricular remodelling and prognosis after acute myocardial infarction. Eur Heart J. 29 (17): 2116-2124. doi: 10.1093/eurheartj/ehn315.

11. Giulia Angelini, Davide Flego, Ramona Vinci, Daniela Pedicino, Francesco Trotta, Aureliano Ruggio, Giuseppe P. Piemontese, Domenico Galante, Myriana Ponzo, Luigi M. Biasucci, Giovanna Liuzzo, Filippo Crea. 2018. Matrix metalloproteinase-9 might affect adaptive immunity in non-ST segment elevation acute coronary syndromes by increasing CD31 cleavage on CD4+ T-cells. Eur. Heart. J. 39(13): 1089-1097. doi: 10.1093/eurheartj/ehx684.

12. Hatem Alfakry, Juha Sinisalo, Susanna Paju, Markku S. Nieminen, Ville Valtonen, Taina Tervahartiala, Pirkko J. Pussinen, Timo Sorsa. 2012. The association of serum neutrophil markers and acute coronary syndrome. Scandinavian Journal of Immunology. 76 (2): 181-187. doi: 10.1111/j.13653083.2012.02718.x.

13. K. J. Molloy, M. M. Thompson, J. L. Jones, E. C. Schwalbe, P. R. F. Bell, A. R .Naylor, I. M. Loftus. 2004. Unstable carotid plaques exhibit raised matrix metalloproteinase-8. Circulation. 110 (3): 337-340 doi: 10.1161/01.CIR.0000135588.65188.14.

14. Khalil A. 2018. Matrix Metalloproteinases, Vascular Remodeling, and Vascular Disease Advances in Pharmacology. 81: 241-330. DOI: 10.1016/bs.apha.2017.08.002.

15. Kristine Y. Deleon-Pennell, Raffaele Altara, Andriy Yabluchanskiy, Alessandra Modesti, Merry L. Lindsey. 2015. The Circular Relationship between Matrix Metalloproteinase (MMP)-9 and Inflammation following Myocardial Infarction. IUBMB Life.67 (8): 611-618. doi: 10.1002/iub.1408.

16. Kristine Y. DeLeon-Pennell, Yuan Tian, Bai Zhang, Courtney A. Cates, Rugmani Padmanabhan Iyer, Presley Cannon, Punit Shah, Paul Aiyetan, Ganesh V. Halade, Yonggang Ma, Elizabeth Flynn, Zhen Zhang, Yu-Fang Jin, Hui Zhang, Merry L. Lindsey. 2016. CD36 is a matrix metalloproteinase-9 substrate that stimulates neutrophil apoptosis and removal during cardiac remodeling. Circ. Cardiovasc. Genet. 9: 14-25.

17. M. Lindsey, K. Wedin, M. D. Brown, C. Keller, A. J. Evans, J. Smolen, A. R. Burns, R. D. Rossen, L. Michael, M. Entman. 2001. Matrix-dependent mechanism of neutrophil-mediated release and activation of matrix metalloproteinase 9 in myocardial ischemia/reperfusion. Circulation. 103:2181-2187.

18. Marta Miguel Turu, Jerzy Krupinski, Esther Catena, Ana Rosell, Joan Montaner, Francisco Rubio, Jose Alvarez-Sabin, Marc Cairols, Lina Badimon. 2006. Intraplaque MMP-8 levels are increased in asymptomatic patients with carotid plaque progression on ultrasound. Atherosclerosis. 187 (1): 161-169. doi: 10.1016/j.atherosclerosis.2005.08.039.

19. Monika Pavkova Goldbergova, Jiri Parenica, Jiri Jarkovsky, Petr Kala, Martin Poloczek, Jan Manousek, Krystyna Kluz, Lenka Kubkova, Simona Littnerova, Martin Tesak, Ondrej Toman, Nikolas Pavek, Zdenka Cermakova, Josef Tomandl, Anna Vasku, Jindrich Spinar. 2012. The Association Between Levels of Tissue Inhibitor of Metalloproteinase-1 with Acute Heart Failure and Left Ventricular Dysfunction in Patients with ST Elevation Myocardial Infarction Treated by Primary Percutaneous Coronary Intervention. Genet. Test. Mol. Biomarkers.16 (10): 1172-1178. doi:10.1089/gtmb.2012.0120.

20. Ohuchi E., Imai K., Fujii Y., Sato H., Seiki M,. Okada Y. 1997. Membrane type 1 matrix metalloproteinase digests interstitial collagens and other extracellular matrix macromolecules. Biol Chem 24; 272(4) 2446-2451. doi: 10.1074/jbc.272.4.2446.

21. Rogelio Zamilpa, Elizabeth F. Lopez, Ying Ann Chiao, Qiuxia Dai, Gladys P. Escobar, Kevin Hakala, Susan T. Weintraub, Merry L. Lindsey. 2010. Proteomic analysis identifies in vivo candidate matrix metalloproteinase-9 substrates in the left ventricle post-myocardial infarction. Proteomics. 10: 2214-2223. 
22. Rogelio Zamilpa, Jessica Ibarra, Lisandra E. de Castro Brás, Trevi A. Ramirez, Nguyen Nguyen, Ganesh V. Halade, Jianhua Zhang, Qiuxia Dai, Tariq Dayah, Ying Ann Chiao, Wesley Lowell, Seema S. Ahuja, Jeanine D’Armiento, Yu-Fang Jin, Merry L. Lindsey. 2012. Transgenic overexpression of matrix metalloproteinase-9 in macrophages attenuates the inflammatory response and improves left ventricular function post-myocardial infarction. J. Mol. Cell. Cardiol. 53: 599-608.

23. Rogelio Zamilpa, Jessica Ibarra, Lisandra E. de Castro Brás, Trevi A. Ramirez, Nguyen Nguyen, Ganesh V. Halade, Jianhua Zhang, Qiuxia Dai, Tariq Dayah, Ying Ann Chiao, Wesley Lowell, Seema S. Ahuja, Jeanine D’Armiento, Yu-Fang Jin, and Merry L. Lindsey, Ph.D. 2012. Transgenic overexpression of matrix metalloproteinase-9 in macrophages attenuates the inflammatory response and improves left ventricular function post-myocardial infarction. J. Mol. Cell. Cardiol. 53: 599-608.

24. Rugmani Padmanabhan Iyer, Mira Jung, Merry L. Lindsey. 2016. MMP-9 signaling in the left ventricle following myocardial infarction. Am. J. Physiol. Heart. Circ. Physiol. 311 (1): 190-198. doi: 10.1152/ajpheart.00243.

25. Takahiko Naruko, Makiko Ueda, Kazuo Haze, Allard C. van der Wal, Chris M. van der Loos, Akira Itoh, Ryushi Komatsu, Yoshihiro Ikura, Masayuki Ogami, Yoshihisa Shimada, Shoichi Ehara, Minoru Yoshiyama, Kazuhide Takeuchi, Junichi Yoshikawa, Anton E. Becker. 2002. Neutrophil infiltration of culprit lesions in acute coronary syndromes. Circulation. 106 (23): 2894-2900. doi: 10.1161/01.cir.0000042674.89762.20.

26. V. Knäuper, G. Murphy, H. Tschesche. 1996. Activation of human neutrophil procollagenase by stromelysin 2. European Journal of Biochemistry. 235 (1-2): 187-191.

27. Yukihiko Momiyama, Reiko Ohmori, Nobukiyo Tanaka, Ryuichi Kato, Hiroaki Taniguchi, Takeshi Adachi, Haruo Nakamura, Fumitaka Ohsuzu. 2010. High plasma levels of matrix metalloproteinase-8 in patients with unstable angina. Atherosclerosis. 209 (1): 206-210. DOI:https://doi.org/10.1016/j.atherosclerosis.2009.07.037.

\section{ИНФОРМАЦИЯ ОБ АВТОРЕ}

Букатов Владислав Владимирович, врачкардиолог палаты реанимации и интенсивной терапии отделения неотложной кардиологии ОГБУЗ «Белгородская областная клиническая больница Святителя Иоасафа», г. Белгород, Россия

\section{INFORMATION ABOUT THE AUTHOR}

Vladislav V. Bukatov, cardiologist of the ward of reanimation and intensive care of the department of emergency cardiology, OGBUZ «Belgorod Regional Clinical Hospital of St. Joasaph», Belgorod, Russia 